\title{
Défis et stratégies d'autonomisation foncière en contexte de changement climatique chez les jeunes du delta du Saloum (Sénégal)
}

\author{
Mamadou Diméa, Marie Fall ${ }^{b}$
}

RÉSUMÉ. Malgré sa riche biodiversité, le delta du Saloum situé au centre-ouest du Sénégal est soumis depuis plusieurs années à différents types de contraintes qui contribuent à la dégradation de son écosystème. Ces contraintes sont amplifiées par le changement climatique dans cette zone classée à haut risque. L'une des conséquences de cette crise est la raréfaction des terres. Le foncier devient une ressource convoitée dans ce contexte insulaire et de fragilité écosystémique. Les communautés et les catégories sociales les plus vulnérables se trouvent exclues de l'accès au foncier pour des motifs économiques et en raison de considérations socioculturelles et religieuses. Cette recherche vise à analyser les défis et les stratégies des jeunes pour accéder au foncier en contrastant leurs aspirations et leurs pratiques.

\begin{abstract}
Despite its rich biodiversity, the Saloum Delta located in West Central Senegal has been subjected for several years to numerous types of constraints that contribute to the degradation of its ecosystem. These constraints are amplified by climate change in an area classified as high risk. One consequence of this crisis is the shortage of arable land. Land is undergoing in the context of fragile ecosystem of high population growth. The most vulnerable persons are not able to access to land. Young people face strong difficulties accessing to land for economic reasons as well as socio-cultural and religious considerations. This research aims documenting the challenges young people face and the strategies they develop in order to access to land by contrasting their expectations and practices.
\end{abstract}

\section{Mise en contexte}

La Réserve de biosphère du delta du Saloum (RBDS) est classée zone humide internationale. Elle est située au centre-ouest du Sénégal et bordée au sud par la Gambie. Actuellement, la RBDS s'étend sur 330000 hectares (ha) dont 76000 ha constituent les ensembles amphibies et maritimes du Parc national du delta du Saloum. Sur le plan administratif, elle fait partie du département de Foundiougne (région de Fatick). La zone hydrographique du delta du Saloum est un estuaire comportant plusieurs bras de mer (le Saloum, le Diomboss et le Bandiala) qui sont interconnectés par un réseau complexe de chenaux de marée connus sous le nom de bolongs (Fall, 2006).
La population du delta du Saloum appartient en majorité aux groupes ethniques des Socés et des Niominkas. Installés dans les îles Bétenti et dans le Niombato, les Socés ont pour principales activités l'agriculture, la pêche et le commerce. La société socé est singularisée par un système de castes et la prégnance d'un système inégalitaire et d'une forte rivalité sociale. Vivant dans les îles Gandoul, les Niominkas qui appartiennent au grand groupe des Sérères, ont la pêche et l'agriculture pluviale comme principales activités. Les Niominkas forment une société égalitaire, sans hiérarchisation sociale et sans système de caste, et qui est fondée sur une intense solidarité horizontale et verticale (Gastellu, 1981).

\footnotetext{
${ }^{a}$ Enseignant-Chercheur, Université Gaston-Berger de Saint-Louis - Sénégal

${ }^{\mathrm{b}}$ Professeure-Chercheure, Université du Québec à Chicoutimi
} 


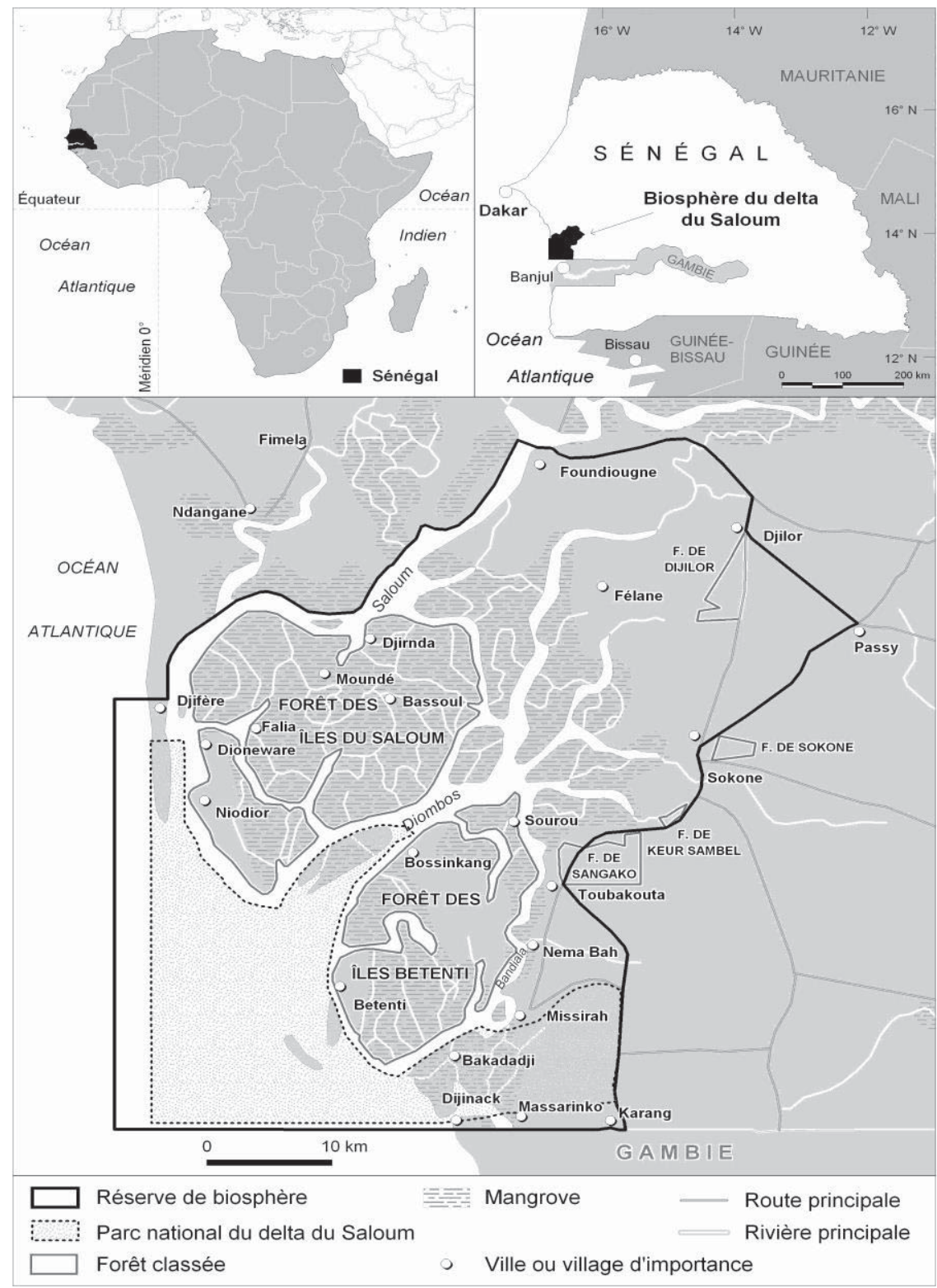

Carte 1 - Localisation géographique du delta du Saloum Source : Fall (2006)

Comme ailleurs en milieu rural sénégalais, la population du delta du Saloum est caractérisée par la prédominance des jeunes. Cette catégorie sociale ne saurait être réduite à son acception démographique. Elle renvoie plutôt à une réalité sociale liée à la prise de rôles dans l'espace domestique et à l'échelle de la société globale. Elle fait donc davantage référence à un manque d'autonomie sur les plans économique, foncier et social. Une des conditions clés de l'accès à l'autonomie, donc du passage de «cadet social» et «d'aide familial » à celui socialement plus prestigieux «d'aîné social », reste l'accès à la ressource foncière. L'accès à la terre est devenu plus ardu pour les jeunes dans un contexte de raréfaction des espaces et de forte pression démographique qui attise la compétition foncière, le tout sur fond de changement climatique qui vient exacerber la convoitise et les conflits autour d'un domaine foncier de plus en plus limité (Bouchard et al., 2010; Niang et al., 2015; Bélières, 2013). 
La migration en ville ou à l'étranger est, ici comme ailleurs en milieu rural sénégalais, la stratégie par excellence pour atteindre l'autonomie économique et la réussite sociale tant recherchées (Villeneuve, 2010). Pour les jeunes qui ne partent pas, il faut miser sur les ressources que leur confèrent leur ouverture vers l'extérieur et leur capacité à décrypter les mutations observables dans leurs villages. Ainsi, dans les villages présentant des attraits touristiques comme Dionewar, certains jeunes recourent à un modèle de "partenariat d'affaires autour du foncier» avec de préférence un toubab ${ }^{1}$ qui apporte les capitaux pour mettre en valeur les ressources foncières qui leur sont octroyées par la collectivité locale. D'autres comptent sur un partage du patrimoine foncier lors du décès du père de famille qui exerce jusque-là sa mainmise sur les ressources foncières du fait d'une conception communautaire de la propriété, des moyens et facteurs de production (Falque et Lamotte, 2008; Kouassigan, 1966; Le Bris et Le Roy, 1983).

Cette contribution s'appuie sur des données recueillies au moyen d'une démarche ethnographique (observation, entretiens individuels semistructurés) pour étudier les défis et les stratégies d'accès au capital foncier chez les jeunes dans le delta du Saloum.

Le delta du Saloum est l'espace de concentration de nos recherches depuis de nombreuses années (Fall, 2006; Dimé et Fall, 2009). Nous mettons à profit nos séjours réguliers dans la zone pour décrypter les mutations écologiques et socio-économiques observables dans les villages constitutifs de cet espace. C'est en étudiant les incidences sociales des changements climatiques dans la zone que la question foncière est apparue à nos yeux comme un enjeu crucial. Comme ailleurs en milieu rural sénégalais, l'accès au foncier est plus contraignant surtout pour les femmes et les jeunes. Nous avons décidé de nous focaliser sur la seconde catégorie pour mettre en lumière les stratégies que les jeunes déploient pour accéder à l'autonomie foncière et les contraintes d'ordres économique, socioculturel et institutionnel rencontrées. Le travail de recherche sur le terrain a été effectué au cours de l'année 2016 et en début 2017. Les trois séjours de recherche que nous avons effectués nous ont permis de mener une série d'observations directes, mais surtout de réaliser des entretiens individuels et de groupe avec des jeunes présentant des profils variés : agriculteurs, pêcheurs, guides touristiques, chômeurs, travailleurs saisonniers, etc.

Les entretiens n'ont pas été limités aux jeunes. En effet, pour avoir une vision plus globale de la question foncière et comprendre ses différents soubassements, ils ont été élargis à des personnesressources formées d'élus locaux (maires, conseillers communaux responsables de la gestion du domaine foncier), à des chefs de village, aux responsables de structures déconcentrées de l'État (service des impôts et domaines, service des eaux et forêts), et aux administrateurs du Parc national du delta du Saloum. L'ensemble des données recueillies et traitées au moment de l'analyse thématique nous permet de décrire les ressources (symboliques, économiques, informationnelles, réticulaires, etc.) mobilisées par les jeunes en vue d'acquérir du capital foncier dans un contexte de changement climatique. Elles permettent également d'analyser les pratiques juvéniles de recherche de l'autonomisation foncière et les multiples significations attribuées à cette aspiration.

\section{Un contexte de fortes turbulences foncières au Sénégal}

Comme bien d'autres pays africains, le Sénégal vit une période de turbulences foncières (Niang et al., 2015; Sy et al., 2013; Koopman, 2012; CONGAD, 2012; Faye, 2011). La terre est non seulement devenue une ressource limitée, mais elle est l'objet de convoitises et de spéculations. Les zones d'habitation ne cessent de grignoter sur les espaces fonciers autour des villes alors qu'en milieu rural, les bouleversements climatiques contribuent fortement à la dégradation des terres cultivables.

Dans ce climat de tensions autour du foncier, les zones rurales sont l'objet d'assauts de plusieurs acteurs. Du fait d'une forte poussée urbaine, effet direct d'une rapide croissance démographique, elles suscitent l'appétit de néocitadins qui, faute de moyens, édifient des quartiers de fortune à la périphérie des villes. En outre, elles aiguisent la boulimie foncière des acteurs de l'agrobusiness. Beaucoup de villes ont quasiment épuisé leurs réserves foncières et continuent de grignoter sur les terres agricoles situées dans les villages avoisinants. À l'instar d'autres pays africains, le Sénégal fait face au 
phénomène d'accaparement des terres (Sy et al., 2013; Faye, 2011).

Dans ce contexte, la terre est devenue « une valeur refuge» et un secteur d'investissement à forte rentabilité financière. Les terres attirent des spéculateurs motivés par un rapide et lucratif retour sur investissement, et des entreprises étrangères qui projettent d'édifier de vastes domaines agricoles ou de s'adonner à d'autres usages comme l'exploitation minière, l'amodiation de zones de chasse ou le développement d'infrastructures touristiques.

Le Sénégal est caractérisé par la cohabitation d'un système dual de gestion et d'exploitation des ressources foncières (Fourchard, 2007; Goislard et Cubrilo, 1998; Chaleard et Mesclier, 2010; Lavigne, 2009; Verdier et Rochegude, 1995). Il s'agit des pratiques coutumières et du droit moderne. La législation foncière est encadrée par la Loi sur le domaine national et par de nombreux autres dispositifs institutionnels ${ }^{2}$. Dans le cadre des politiques de décentralisation, l'État a transféré aux collectivités locales (communes urbaines, communes rurales) ${ }^{3}$ un certain nombre de responsabilités parmi lesquelles celle très cruciale de la gestion foncière. La prégnance des régimes coutumiers de dévolution des terres, malgré les ruptures que l'État a voulu introduire sur les plans législatif et réglementaire, est de nature à perpétuer et accentuer les inégalités autour du foncier et à aggraver les difficultés d'accès au foncier des jeunes. Ceux-ci, dans un contexte de rétrécissement de l'espace, peuvent se trouver en marge des compétitions foncières ou n'accéder qu'aux terres les moins convoitées, car moins fertiles ou éloignées des points d'eau ou se situant dans des espaces dégradés à cause de phénomènes comme la salinisation, l'ensablement ou le déboisement.

Un autre phénomène concourant à la récurrence des tensions est la dégradation des sols et le changement climatique. Les événements climatiques extrêmes auxquels est associé le changement climatique (inondations, sécheresses, élévation du niveau de la mer) représentent des facteurs de baisse de fertilité de l'espace cultivable ainsi que de son amenuisement. Toutes les zones agroécologiques constitutives de l'espace national sont exposées à ces menaces, mais elles se retrouvent plus accentuées dans les zones côtières comme le delta du Saloum où la destruction des écosystèmes estuariens de la zone se conjugue avec une salinisation des terres de culture. Cela a pour conséquence une diminution de l'espace et du même coup une exacerbation des situations d'exclusion foncière frappant les jeunes.

Malgré leur poids démographique et leur place prépondérante dans les exploitations familiales comme force de travail (ANDS, 2014), les jeunes ruraux continuent de buter sur de nombreux obstacles pour obtenir de la terre. Ce qui a pour conséquence de les confiner dans une précarité socioéconomique et professionnelle. Dans le cadre d'une étude (Niang et al., 2015) menée dans le delta du fleuve Sénégal et dans la zone des Niayes, localités recelant de réelles potentialités agricoles, les jeunes accèdent à la ressource foncière essentiellement de deux manières : l'héritage et la location. Ce qui freine leurs capacités de production et leur autonomie.

Les prescriptions sociales et les normes religieuses contribuent fortement au fait que les jeunes possèdent peu de pouvoir social et décisionnel pour espérer influer les processus et les règles d'affectation et de désaffection des terres, car ils sont peu représentés dans les organes délibérants, les commissions domaniales et les instances villageoises. Ces structures sont les lieux où se prennent les décisions relatives aux questions foncières. Les jeunes ont certes de fortes aspirations à la réussite sociale, mais celles-ci sont contrecarrées par leur faible autonomie. Certains jeunes arrivent néanmoins à déployer des stratégies alternatives qui passent souvent par l'abandon des activités agricoles et l'investissement de la filière commerciale, la transformation des produits agricoles ou la réalisation d'initiatives entrepreneuriales (transformation de produits agricoles, prestations de services, etc.). Le portrait que Niang et al. (2015) brossent des jeunes dans le secteur de l'agriculture est quasiment partagé par la majorité des jeunes en milieu rural sénégalais. Les jeunes du delta du Saloum font face à des entraves de nature analogue pour accéder à la terre.

\section{Le delta du Saloum : un écosystème fragilisé et des ressources foncières limitées}

La Réserve de biosphère du delta du Saloum fait partie du patrimoine mondial de l'UNESCO. À ce titre, elle doit remplir les fonctions de conservation en système ouvert, soit la protection des écosystèmes à l'état naturel, le maintien de la diversité écologique et de 
l'équilibre du milieu naturel, et la conservation des ressources génétiques. Elle doit assurer les fonctions de recherche et de protection de l'environnement, les fonctions d'éducation et de formation, et les fonctions de coopération pour le développement (Fall, 2006; Sarr, 2013). La réserve est subdivisée en deux zones. L'aire centrale correspond au Parc national du delta du Saloum où les écosystèmes sont entièrement protégés contre toute exploitation. Sa spécificité réside dans le fait qu'il héberge le village Bakadadji. L'aire périphérique appelée aussi aire à usages multiples correspond aux terroirs villageois dont certains sont contigus au parc, notamment Missirah, Djinack, Massarinko, Betenti, Samé, Karang et Karamba. Une troisième zone qualifiée de zone tampon entre l'aire centrale et l'aire périphérique reste toujours à définir (Fall, 2006; Fall et Villeneuve, 2010).

Sur le plan géographique, la réserve de biosphère forme un écosystème côtier et marin qui s'ouvre sur l'Océan Atlantique. Le Parc national du delta du Saloum, créé en 1976, occupe une partie du domaine estuarien, une partie du domaine maritime et la frange côtière du domaine continental. Cette aire aux fortes potentialités naturelles a été fusionnée à l'ensemble du complexe estuarien en 1981 pour former la Réserve de biosphère du delta du Saloum, site du patrimoine mondial de l'UNESCO. En 1984, cette zone d'accueil de plusieurs espèces d'oiseaux paléarctiques lui a valu d'être classée « zone humide d'importance internationale» en vertu de la Convention sur les zones humides signée à Ramsar (Fall, 2006).

Le delta du Saloum est le premier site de biodiversité du Sénégal. Son statut de réserve de biosphère est destiné à protéger les ressources naturelles de la zone en créant des activités de développement qui pourraient sauvegarder la biodiversité en fixant des objectifs fondés sur des connaissances et une planification appropriée (Féral, 2007).

L'importance écologique et économique de cet espace se lit à travers la diversité de ses milieux naturels. Sa situation dans la zone de transition entre le domaine soudano-sahélien au nord et le domaine soudano-guinéen au sud lui confère une richesse floristique et une diversité de formations forestières (Fall, 2006), sans compter toute la dynamique sociale et économique qui résulte de sa position de carrefour entre le Sénégal et la Gambie, entre les régions de Fatick et de Kaolack, ainsi qu'entre plusieurs arrondissements.
Au Sénégal, le delta du Saloum est classé parmi les sites à risque par rapport au changement climatique (Sarr, 2013; Sy, 2012; Dimé, 2017). Le climat de la zone est soumis à des modifications de taille, à l'instar de presque tout le Sénégal. Depuis plusieurs décennies, la pluviométrie connait une tendance à la baisse. Si la quantité moyenne d'eau était de 776,63 millimètres entre 1941 et 1970, elle ne dépassait guère les 555 millimètres entre 1971 et 2000 (Villeneuve, 2010; Fall, 2006). La diminution des précipitations a de multiples conséquences. Elle affecte les rendements agricoles, entraîne la baisse de la disponibilité d'eau douce tout en contribuant à l'avancée des tann, ces étendues de terre dénudées et à forte teneur en sel et impropres à l'agriculture. La végétation de mangrove qui a un rôle écologique et économique très important se réduit du fait des changements climatiques et de sa forte exploitation. Un autre facteur important à la dégradation de l'écosystème du delta du Saloum est lié aux actions anthropiques. L'exploitation abusive et incontrôlée peut prendre diverses formes: surpâturage, agriculture extensive sur brûlis, déboisement, utilisation de filets non réglementaires pour la pèche, braconnage, etc. (Dimé, 2017).

La singularité de l'écosystème du delta du Saloum explique globalement la faiblesse des ressources foncières disponibles pour les activités agricoles. Donc, on est ici dans un contexte où les disponibilités foncières sont limitées. Cette faiblesse des espaces est aiguisée par la dégradation de l'environnement à laquelle est en proie la région. Comme ailleurs au Sénégal, dans le delta du Saloum les modes de gouvernance foncière font intervenir une pluralité de références.

\section{La diversité des modes de gouvernance foncière et des corpus réglementaires}

\subsection{Le droit coutumier en gestion des ressources}

Dans les sociétés traditionnelles socé et niominka, la parole est un moyen fondamental d'organisation des rapports interindividuels. Le détenteur de tous les droits territoriaux est le chef de terre qui est défini comme le premier occupant de l'espace, sa descendance, et parfois le nouvel arrivant qui a fait alliance avec lui. Il est de ce fait le symbole vivant de l'alliance sacrificielle contractée avec la terre et toutes ses ressources. Le droit coutumier en gestion 
des ressources est davantage axé sur les terres. Selon l'explication reçue de l'Alcalis du village de Betenti, quand la terre leur appartient, tout ce qui y vit comme les forêts et les animaux, et tout ce qui la borde comme les cours d'eau est à son propriétaire. Ainsi, dans les sociétés de pêcheurs comme la société niominka, l'espace des bolongs qui bordent les villages appartient aux villageois. Dans le droit coutumier relatif à la gestion des ressources, nous avons le droit du premier occupant, le droit de feu et de hache ainsi que le droit de succession.

\subsection{Le droit du premier occupant}

De l'avis des chefs des villages de Missirah, Niodior et Néma Bah, le droit du premier occupant stipule qu'a priori la terre et les ressources qu'elle englobe appartiennent au premier occupant, c'est-à-dire à celui qui les découvre et qui s'y implante en premier. Ainsi, il a tous les droits d'occuper l'espace et d'en exploiter les ressources forestières, maritimes et terrestres. C'est lui qui légifère, prend les décisions sur la gestion et exerce une autorité sur les membres du groupe établi sur sa terre et l'espace territorial.

\subsection{Le droit de feu et de hache}

Ce droit est en vigueur dans la gestion des terres de culture. Les terroirs villageois actuels étaient d'abord des territoires forestiers. Les premiers à s'y être installé ont dû brûler la forêt ou couper les arbres à l'aide d'une hache pour avoir des terres où habiter et pratiquer des activités agricoles. D'où les appellations de « droit de feu » et de «droit de hache». Dans les villages du Saloum, les familles dont les ancêtres ont autrefois déblayé le terrain se disent aujourd'hui détentrices de tous les droits dans la gestion des ressources et surtout prioritaires quant à leur distribution. Les descendants de ces premiers occupants sont les Lamanes ou maîtres des terres.

\subsection{Le droit de succession}

Ce droit est appliqué dans le cas où le propriétaire de la terre ou le détenteur des droits d'occupation et d'exploitation des ressources terrestres et maritimes meurt ou renonce à ses droits. Ainsi, ses droits reviennent à sa descendance. Par exemple, les champs se lèguent de père en fils en société patrilinéaire et d'oncle à neveu en société matrilinéaire. Le domaine forestier du père passe entre les mains des fils et les zones de pêche de l'oncle au neveu dépendamment de la structure sociale. Dans les sociétés traditionnelles, la terre appartient à ceux qui l'ont découverte. Le détenteur de tous les droits ancestraux est le Lamane. Il est actuellement défini dans les sociétés niominka et socé comme un citoyen chargé d'un service et protégé à ce titre. Par ailleurs, le Lamane est chargé personnellement au titre de ses capacités et par référence à une attribution personnelle de gérer l'espace villageois et ses alentours.

Le droit coutumier favorise la "parentalisation» des individus et les ressources sont accessibles par tous les membres de la communauté. En accord avec Leroy (1979), nous confirmons que ressources et parenté agissent l'un sur l'autre en ce sens que la ressource se "parentalise » et que la parenté se «territorialise». Cependant, aucune loi écrite ne rend compte de cette interaction; nous en concluons que c'est parce que le droit coutumier est caractérisé par son oralité qui ne se réfère pas simplement à certains moyens spécifiques de consultation des sources, contrairement au droit moderne avec les articles de loi. La principale contrainte par rapport au droit coutumier réside dans l'absence de sources écrites consultables à ce sujet.

\subsection{Le corpus moderne en gestion des ressources}

Le régime juridique moderne organisant la gestion des ressources naturelles dans la Réserve de biosphère du delta du Saloum repose essentiellement sur l'application des lois et décrets d'application relatifs à la gestion environnementale. Il s'agit de la Loi sur le domaine national, le Code du domaine de l'État, le Code forestier, le Code de la chasse et de la protection de la faune, le Code des collectivités locales, le Code de l'environnement, le Code de la pêche maritime, le Décret portant organisation des parcours du bétail et fixant les conditions d'utilisation des pâturages, et les décrets d'application de ces lois4.

\subsubsection{La Loi sur le domaine national}

La Loi no 64-66 du 17 juin 1964 relative au domaine national ${ }^{5}$ ainsi que les différents textes juridiques relatifs à son application organisent le régime juridique des terres du domaine national : Décret $n^{\circ} 72-1288$ relatif aux conditions d'affectation et de désaffectation des terres du domaine national comprises dans 
les communautés rurales, Décret n ${ }^{\circ}$ 64-573 fixant les conditions d'application de la loi relative au domaine national, Décret $n^{\circ}$ 64-574 portant application de l'article 3 de la Loi autorisant à titre transitoire l'immatriculation au nom des occupants ayant réalisé une mise en valeur à caractère permanent. Différentes questions sont ainsi réglementées, notamment la classification des terres du domaine national dans l'article 4, les conditions d'affectation et de désaffectation des terres, l'organisation administrative et la gestion du domaine national, et les droits des occupants des terres.

\subsubsection{La Loi sur le transfert des compétences}

Considérée comme "l'Acte 2 de la décentralisation », la Loi no 96-07 du 22 mars 1996 portant transfert des compétences aux régions, aux communes et aux communautés rurales ${ }^{6}$ renforce les compétences des collectivités locales en matière de gestion des terroirs. Elle donne davantage de prérogatives aux collectivités locales en matière de gestion du domaine national, notamment des zones de terroir. Depuis 2013, une nouvelle étape a été franchie dans les politiques de décentralisation au Sénégal avec la mise en œuvre d'une nouvelle loi régissant les collectivités locales et qui est communément appelée « l'Acte 3 de la décentralisation ». En effet, la Loi $n^{\circ}$ 2013-10 du 28 décembre 2013 portant Code général des Collectivités locales ${ }^{7}$ consacrent une communalisation intégrale, l'érection des départements comme collectivités locales à la place des régions et enfin l'établissement de pôles territoriaux de développement. L'objectif est de parvenir à une territorialisation des politiques publiques et à une responsabilisation accrue des décideurs locaux et à plus de proximité dans la gestion des affaires publiques notamment dans la gestion foncière.

\subsubsection{Les autres dispositifs institutionnels réglementaires}

Le Code du domaine de l'État réglemente les propriétés de l'État et dont la gestion relève de son domaine de compétence. Ainsi, certains articles ont trait directement aux ressources naturelles, et leur utilisation en terroir villageois ne peut se faire sans autorisation. La Loi no 1998-03 du 20 février 1998 portant Code forestier $^{8}$ et la Loi n ${ }^{\circ} 86-04$ du 24 janvier 1986 portant Code de la Chasse et de la Protection de la Faune sont les références juridiques des agents de l'administration forestière notamment ceux des parcs nationaux et des eaux et forêts. Ces codes régissent la gestion des forêts, des espaces protégés et du PNDS. La Loi n ${ }^{\circ} 83-$ 05 portant Code de l'environnement réglemente la surveillance et la gestion de l'environnement et fixe les règles de base en matière de protection de l'environnement. Il stipule en son chapitre 2, article L3 qu'il a pour objet d'établir les principes fondamentaux destinés à gérer, à protéger l'environnement contre toutes les formes de dégradation, afin de valoriser rationnellement l'exploitation des ressources naturelles, de lutter contre les différentes sortes de pollutions et nuisances et d'améliorer les conditions de vie des populations dans le respect de l'équilibre de leurs relations avec le milieu ambiant. La Loi no 2015-18 du 13 juillet 2015 portant Code de la pêche maritime réglemente ainsi l'accès aux zones de pêche et les modalités de l'exploitation des espèces halieutiques. Le décret d'application de la loi précise le maillage des filets autorisés et l'usage des engins. Le Décret portant organisation du bétail fixe les conditions d'utilisation des pâturages, réglemente les ressources pastorales et organise les parcours de bétail en définissant les conditions d'utilisation des pâturages et des points d'eau.

\section{Les stratégies juvéniles d'accès à la terre dans le delta du Saloum}

Les jeunes du delta du Saloum ne se trouvent pas dans une situation où ils sont totalement démunis sur le plan foncier. Nos enquêtes de terrain ont permis de nous rendre compte que certains d'entre eux possédaient de la terre, mais peu ou faiblement valorisée. Tous ont conscience que la terre est devenue aujourd'hui un des biens les plus précieux, car de plus en plus rare. Elle est un secteur d'investissement des plus rentables. Certains jeunes ont souligné qu'il n'en a pas toujours été ainsi.

La prise de conscience de la valeur de la terre, surtout de sa valeur marchande, s'est opérée récemment dans le delta du Saloum. La perception prédominante était que dans les villages du delta du Saloum, les populations disposaient de terres en abondance et suffisamment arrosées pour permettre le développement d'une agriculture de subsistance. La pratique de la pêche et la cueillette de fruits de mer complétaient la gamme des activités socio-économiques. C'est à partir de la création du Parc national du delta du Saloum que la situation a commencé à changer. L'aire centrale et 
la zone tampon du parc relèvent de l'administration du parc; en conséquence, les populations perdent la jouissance de toutes les ressources s'y trouvant. L'aire centrale correspond au Parc national du delta du Saloum où les écosystèmes sont entièrement protégés contre toute exploitation. Sa spécificité réside dans le fait qu'il héberge le village de Bakadadji. L'aire périphérique appelée aussi aire à usages multiples correspond aux terroirs villageois dont certains sont contigus au parc, notamment Missirah, Djinack, Massarinko, Betenti, Samé, Karang et Karamba. Une troisième zone qualifiée de zone tampon entre l'aire centrale et l'aire périphérique reste toujours à définir. Le Parc national du delta du Saloum a été créé en 1976 dans la controverse.

En effet, pendant la période coloniale, le gouverneur Dupuis, en classant la forêt de Fathala en 1935 (Décret 1688/SE du 20 juillet 1935), avait promis aux populations des villages riverains que ce serait la dernière fois qu'elles seraient privées de leurs terres. Elles auraient le droit de cultiver et d'utiliser les ressources des terres non classées. Mais en 1976, quand l'État a voulu encore confisquer des terres du terroir villageois et les joindre à une partie de la forêt classée de Fathala pour en faire un parc national, les populations ont refusé de céder leurs terres et de perdre leurs droits sur les ressources. Des villages entiers devaient être déguerpis pour faire place au PNDS.

Finalement, la raison d'État a prévalu malgré de multiples refus des populations. Les ruptures induites par l'érection du parc, dans les perceptions de l'espace et dans la délimitation des terroirs villageois, ont entraîné de facto un renversement du regard porté sur la ressource foncière. Les populations faisaient face à un choc des légitimités entre celle coutumière héritée de pratiques ancestrales et des traditions et celle institutionnelle portée par l'État et ses services techniques (Eaux, forêts et chasse, Environnement et établissements classés) jugée plus puissante (Fall, 2006).

L'établissement du parc s'est fait dans un contexte où les écosystèmes du delta du Saloum subissaient les premiers effets de la baisse pluviométrique ainsi que les conséquences d'une exploitation abusive des ressources forestières, halieutiques et hydriques. Au même moment, les populations ne cessaient d'augmenter, effet direct d'une croissance démographique très rapide comme partout en milieu rural sénégalais. L'espace foncier disponible rétrécissait tandis que les demandeurs, surtout ceux de la frange juvénile, devenaient plus nombreux. La terre devient un bien marchand dont la valeur ne cesse de grimper. Les populations semblent plus conscientes qu'il est possible de s'enrichir ou de réaliser des projets à partir des ressources foncières dont elles disposaient.

De l'avis d'un jeune interrogé à Missirah, la ruée vers la terre a été accélérée par l'arrivée de certains résidants aisés des villes. Ces derniers sont surtout attirés par les opportunités socio-économiques liées au développement d'activités touristiques et à la pratique de l'arboriculture fruitière ainsi que par le coût modique ou la gratuité de la terre dans les Iles du Saloum comparativement à d'autres localités du pays. Autrefois pratique peu répandue, la course vers la terre et la vente de terre s'est ainsi amplifiée dans les villages. Beaucoup de jeunes ont profité de cet environnement de spéculation foncière pour vendre les terres reçues en héritage afin de financer un projet d'émigration en Europe ou se lancer dans le commerce ou bien migrer en ville. Par exemple, ces dernières années, plusieurs jeunes des villages de Missirah, Betenti, Niodior ou Dionewar ont rejoint dans un premier temps Dakar, la capitale sénégalaise, avant d'effectuer une migration vers la France ou bien l'Espagne.

Cette propension à ne percevoir la terre que par rapport à sa valeur marchande ne se retrouve pas dans tout l'espace du delta du Saloum. Des différences notables subsistent entre les terroirs des Niominkas et ceux des Socés. Ainsi, la partie habitée par les Niominkas est composée essentiellement d'îles (Dionewar, Niodior, Falia). Le caractère communautaire de la terre y est plus prononcé et les disponibilités foncières plus limitées. La vente de terre y est un phénomène relativement inconnu et peu encouragé par les populations. Elles ont conscience de l'étroitesse des surfaces disponibles rognées par l'avancée de la mer. La terre continue d'être une propriété familiale et sa gestion soumise à l'autorité des conseils de famille et de la collectivité locale (conseil rural avant la réforme territoriale, et maintenant la commune).

Dans les îles habitées par les Niominkas, c'est comme si les élus et les populations étaient échaudés par les possibles ravages d'une course à la spéculation foncière dans des domaines insulaires très limités et victimes des contrecoups des changements climatiques (dégâts causés aux berges par l'avancée de la mer). Un jeune interrogé à Dionewar le reconnait clairement : 


\section{Le foncier est très limité à Dionewar. On n'a pas des espaces aussi vastes comme dans la partie continentale du delta. On est obligés d'être vigilants dans la gestion des terres et de prendre des mesures pour ne pas encou- rager que la terre soit juste dans les mains des nantis et que les autres en soient exclus. La terre doit être réservée en priorité aux fils de Dionewar on à des "étrangers» qui ont rendu de précieux services aux populations. Beaucoup de nos terres ont été englouties par la mer et comme nous n'en avons pas assez, il faut être prudent dans son utilisation.}

Tout jeune désireux d'obtenir une terre pour la mettre en valeur aux fins d'habitat ou pour tout autre projet économique peut l'obtenir à même le patrimoine familial ou effectuer une demande auprès de la commune. Celle-ci peut procéder à des lotissements. Mais lorsque ces lotissements se font sur des terres relevant de la propriété d'une famille, les membres ont un quota dans les parcelles loties. Même dans le cas de nouveaux lotissements, la commune met en place des balises en exigeant des attributaires qu'ils entreprennent immédiatement des travaux pour matérialiser leur désir d'habiter sur le site (début de construction, matériaux de construction). Cette exigence est motivée par le désir de refréner toute velléité de spéculation foncière.

Le principal mode d'acquisition foncière pour les jeunes, mentionné à l'unanimité dans nos entretiens, reste l'héritage. C'est souvent à la suite du décès du père de famille qui concentrait dans ses mains la totalité des moyens de production que, comme jeune, on accède finalement à la terre, selon les principes de l'héritage codifié et organisé conformément aux préceptes du droit musulman. Le fait notable à signaler ici est que la fille reçoit la moitié de la part dont hérite le garçon. Peu de jeunes peuvent revendiquer un autre moyen d'obtention de la terre. En dehors de jeunes ayant socialement réussi grâce à l'émigration en Europe et qui ont pu acheter du foncier, l'écrasante majorité des jeunes disposant d'une terre dans les villages du delta du Saloum l'ont reçue en héritage. Pour la plupart, les superficies ne sont guère importantes, car on est ici dans le contexte de sociétés où l'on enregistre un taux élevé de polygamie et où les biens fonciers laissés par le défunt doivent être partagés avec une fratrie nombreuse comme le souligne un jeune interviewé à Nodior :
Quand tu dois partager un terrain de $30 \mathrm{~m}$ sur $30 \mathrm{~m}$ $\left(900 \mathrm{~m}^{2}\right)$ avec 15 frères et scurs, il n'est pas évident de se projeter comme propriétaire d'un petit lopin de terre où aucune activité rentable ne peut être pratiquée.

Certains jeunes ont pu néanmoins obtenir des terrains en suivant la procédure de l'affectation par le conseil rural, instance qui assume la gestion foncière à l'échelle des villages. Afin de voir leur demande couronnée de succès, certains jeunes ciblent des terres situées sur des espaces moins convoités. C'est le cas de deux jeunes de Missirah qui ont créé l'association Fannabara vouée au développement de pratiques d'écocitoyenneté et de l'écotourisme. Cette association est aussi le réceptacle d'initiatives de coopération internationale et accueille depuis neuf ans des étudiants de l'Université du Québec à Chicoutimi dans le cadre d'un programme de stage humanitaire (Dimé et Fall, 2009). Le fait que la demande ait été faite dans le cadre associatif a accru ses chances de réussite, car d'autres jeunes ayant introduit des procédures d'attribution foncière dans le cadre d'une démarche individuelle ont eu moins de succès. L'autre modalité d'accès à la terre est l'emprunt. Faute de terres, les jeunes peuvent disposer provisoirement de lots pour pratiquer l'agriculture. La mise en valeur prend fin dès que le possesseur légitime reprend son bien. La location de terres, phénomène répandu dans des zones comme les Niayes, n'est pas une pratique courante dans le delta du Saloum.

Comme nous l'avons souligné plus haut, la modalité d'accès qui est revenue de manière récurrente dans les discours demeure l'héritage. Mais celui-ci a comme contrecoup de contribuer à un morcellement accentué du patrimoine foncier. Cependant, il convient de souligner que la pratique du morcellement à des fins d'héritage est davantage développée chez les populations appartenant au groupe ethnique des Socés. On est ici dans le contexte d'une société fortement marquée par le patriarcat et d'un esprit de rivalité sociale plus exacerbé. Chez les Niominkas où la base sociale est plus marquée par un égalitarisme socioéconomique, la propriété familiale de la terre continue d'être sauvegardée sur le plan symbolique comme dans les pratiques sociales. Même le mode d'habitat dans les terroirs villageois reflète ce désir de perpétuer cet éthos communautaire, car les quartiers sont établis en fonction des familles. Les parcelles de culture demeurent une propriété familiale et la terre uniquement réservée aux ressortissants des villages. 


\section{Le « business du partenariat » : stratégies juvéniles de valorisation du foncier}

La situation du foncier est contrastée dans le delta du Saloum. L'accès des jeunes à la terre n'est pas uniforme selon qu'on est dans la partie insulaire ou continentale ou en fonction du groupe ethnique dominant. Diverses stratégies de valorisation du foncier peuvent être notées chez les jeunes. La plus notable reste le modèle du partenariat d'affaires avec un expatrié occidental qui apporte des capitaux et le jeune du delta la terre. De l'avis d'un jeune du village de Dionewar qui a été l'un des pionniers de ce type de collaboration, il a réussi à constituer un groupement d'intérêt économique (GIE) avec quatre autres de ses pairs. Ils ont effectué les démarches auprès du conseil rural pour l'enregistrement formel de leur structure jusqu'à l'obtention de leur agrément auprès de la Chambre de commerce, d'industrie et d'agriculture de Fatick. Ensuite, ils ont élaboré un projet de développement de l'apiculture en collaboration avec un Français qui venait séjourner régulièrement à Dionewar et avec qui ils avaient développé des affinités et une relation de confiance. Ce dernier possédait des compétences et avait un intérêt pour l'apiculture.

Sitôt le projet élaboré, les jeunes ont introduit auprès du conseil rural ${ }^{9}$ une demande d'attribution d'une parcelle qui a été acceptée du fait du caractère original du projet et de ses retombées socio-économiques. Sitôt la parcelle attribuée, ils l'ont présentée comme leur apport dans le modèle d'affaires conclu avec le Français qui a apporté l'argent nécessaire pour ériger un bâtiment composé d'un bloc d'habitations et d'un autre utilisé pour la miellerie. Le premier bloc est utilisé comme logement par le Français pendant ses séjours à Dionewar. En son absence, le bâtiment est rentabilisé par les jeunes à travers un système de location courte durée pour des touristes et des visiteurs.

Ce modèle de partenariat a fait tache d'huile auprès des jeunes de Dionewar d'où la multiplication de projets similaires. Certains se sont associés avec leur conjoint(e), d'autres avec des touristes occidentaux réguliers pour édifier des campements comme logement personnel ou locatif. Chez les Occidentaux, les motivations tournent autour de raisons humanitaires et compassionnelles (soutien à de jeunes désœuvrés dans des territoires pauvres), de confort personnel, voire pécuniaires (disposer d'un pied à terre pendant les vacances).
Ainsi, 8 à 12 projets de campements touristiques ont été réalisés à Dionewar dans le cadre de partenariats entre Français qui viennent régulièrement dans les îles et jeunes du village. Le développement de telles initiatives à Dionewar n'est pas surprenant. Ce village présente beaucoup d'attraits touristiques et a abrité un lodge qui a joué un rôle socio-économique important tout en contribuant au rayonnement du village. Il s'agit du delta Niominka, mais qui est cependant fermé depuis $2014^{10}$. La plupart des projets de partenariat portent sur les campements touristiques étant donné les besoins dans ce secteur.

\section{Conclusion}

Globalement, le delta du Saloum présente une singularité quant à l'accès au foncier. On est ici dans le contexte d'une localité où les disponibilités foncières sont limitées par la configuration de l'écosystème. Néanmoins, les populations sont toujours arrivées à faire prévaloir des mécanismes de gestion ayant permis l'absence de conflits majeurs dans la zone contrairement à d'autres localités du Sénégal où les problèmes fonciers suscitent leur lot de tension, voire de violence. Cela ne signifie pas que le phénomène de l'exclusion foncière est inconnu dans le delta du Saloum. Nous avons montré comment les jeunes y étaient exposés et les stratégies qu'ils mettent en place pour accéder à la terre tout comme les usages qu'ils en font pour rentabiliser les terres qui leur sont attribuées. Si certains s'orientent vers une mise en valeur agricole, d'autres en font un usage marchand pour financer des projets d'autonomisation personnelle à travers l'émigration ou pour développer des relations d'affaires autour de projets touristiques.

Dans une localité où les revenus s'acquièrent pour l'essentiel à travers les activités agricoles, il est clair que l'accès à la terre représente une dimension fondamentale dans les dynamiques d'autonomisation socioéconomique des jeunes. Dans les politiques publiques, les jeunes ruraux n'ont pas encore attiré l'attention des décideurs à la mesure de l'intensité de leur précarité socioprofessionnelle inhérente à la situation de dépendance socio-économique dans laquelle la majorité d'entre eux est plongée. Dans le delta du Saloum, le chômage et le sous-emploi des jeunes sont des problèmes préoccupants à l'instar de la majorité des jeunes en milieu rural.

Aujourd'hui, les pouvoirs publics semblent mesurer l'ampleur de ce défi. Cela s'est traduit par la mise en place d'initiatives prônant le retour vers l'agriculture 
et la promotion des activités entrepreneuriales des jeunes ruraux au moyen de programmes comme les Domaines agricoles communautaires (DAC) et des projets de jeunes financés par l'Agence nationale pour l'insertion et le développement agricole (ANIDA) et l'Agence nationale pour la promotion de l'emploi des jeunes (ANPEJ). Mais les jeunes rencontrés dans le delta du Saloum disent ne pas encore bénéficier de l'appui de ces programmes étatiques.

Globalement, les jeunes, malgré leur poids démographique, continuent d'être en position de vulnérabilité sur le plan foncier. Les prescriptions et les normes culturelles dominantes sont souvent à leur désavantage tout comme pour les femmes. Associé à d'autres facteurs socio-économiques comme l'étroitesse du marché du travail en zone rurale et l'accès limité au crédit, le manque de contrôle sur le foncier est un obstacle rédhibitoire à l'autonomisation des jeunes. De ce fait, tout programme désireux de contribuer à la promotion socio-économique de ces jeunes devrait veiller à trouver les moyens d'adapter les modes de tenure coutumière et de contribuer à une plus grande équité générationnelle dans l'accès à la terre.

\section{NOTES}

1 Toubab désigne en langue wolof un Blanc. Ce terme restitue mieux la charge symbolique attachée à la personne blanche dans les imaginaires des populations locales pour qui toubab est synonyme de personne nantie et capable d'apporter des bienfaits (argent, opportunités économiques, projets de développement, savoir-faire) dans des zones où les populations soutiennent avoir des potentialités mais sans moyens (financiers, techniques et matériels) pour les exploiter.

2 Un projet de réforme de cette loi est en cours. Une Commission nationale de la réforme foncière (CNRF) a été mise sur pied en 2014 pour faire un diagnostic exhaustif de la situation foncière et formuler un ensemble de propositions pour « améliorer la gestion foncière pour répondre aux besoins de développement et de cohésion sociale » (repéré à www.cnrf.sn). Les pouvoirs publics semblent opter pour la prudence et la recherche du maximum de consensus étant donné le caractère hautement explosif des enjeux fonciers au Sénégal. La CNRF a débouché sur un document de politique foncière toujours en attente d'être officiellement validé et mis en œuvre par les pouvoirs publics.

3 Le Sénégal a connu depuis son indépendance plusieurs réformes majeures dans le cadre de la décentralisation. On est aujourd'hui dans la troisième phase surnommée Acte 3 de la décentralisation qui consacre le département comme collectivité locale et une communalisation intégrale. Il y a eu en 1996 une réforme majeure qui a conduit à la naissance des régions et au transfert de neuf domaines de compétence par l'État vers les collectivités locales (conseils ruraux, communes) et celle de 1972 qui a donné naissance aux communautés rurales.

4 Les lois et décrets relatifs à la gestion environnementale ont été étudiés et interprétés article par article dans le but de faire ressortir les éléments qui nous renseignaient le mieux sur l'application des règlements dans les aires protégées.

5 Pour plus de détails, voir : http://www.au-senegal.com/IMG/pdf/snarga_loi_nc991.pdf, repéré le 03 septembre 2018.

6 Voir http://www.servicepublic.gouv.sn/assets/textes/loi-transfert-region.pdf, repéré le 3 septembre 2018.

7 Version intégrale de la Loi à l'adresse http:/ / www.jo.gouv.sn/spip.php?article10120, repéré le 20 juin 2018.

8 http://www.droit-afrique.com/upload/doc/senegal/Senegal-Code-1998-forestier.pdf, repéré le 30 août 2018. Un nouveau Code forestier a été adopté le 24 janvier 2018 par le Conseil des ministres. Les principales nouveautés sont dans le durcissement des peines pour les infractions liées à l'exploitation forestière.

9 La communalisation intégrale qui est entrée en vigueur en 2013 avec ce qui est communément appelé l'Acte 3 de la décentralisation a consacré la disparition du conseil rural qui était jusqu'alors l'organe doté de la compétence foncière à l'échelle des communautés rurales.

10 Les propriétaires du delta Niominka ont invoqué diverses raisons pour justifier sa fermeture : le coût élevé des charges (cherté des taxes touristiques), le coût élevé d'approvisionnement en eau et en électricité, l'accès difficile à la zone reliée à l'époque à la ville de Joal par une route cahoteuse en latérite, la baisse des fréquentations consécutive, entre autres, à l'instauration d'un visa d'entrée au Sénégal et à l'épidémie d'Ebola.

\section{RÉFÉRENCES}

Agence nationale de la statistique et de la démographie (2014). Rapport définitif. RGPHAE 2013. Dakar, Sénégal : Ministère de l’Économie, des Finances et du Plan.

Bélières, J-F., Jamin, J-Y., Seck, S. M., Tonneau, J-P., Adamczewski, A. et Gal, P-Y. (2013). Dynamiques foncières, investissements et modèles de production pour l'irrigation en Afrique de l'Ouest : logiques financières contre cohérences sociales? Cabiers Agricultures, 22(31), 61-66. 
Bouchard, C., Marrou, L., Plante, S., Payet, R. et Duchemin, E. (2010). Les petits États et territoires insulaires face aux changements climatiques : vulnérabilité, adaptation et développement. VertigO - la revue électronique en sciences de l'environnement, 10(3). Repéré à http://vertigo.revues.org/10634

Chaleard, J. L. et Mesclier, E. (2010). Introduction. Question foncière et dynamiques territoriales dans les pays du Sud : nouveaux liens, nouvelles approches. Annales de la géographie, (676), 587-596.

Charmes, E. (2007). Le malthusianisme foncier. Études foncières, (125), 12-16.

CONGAD (2012). Rapport de l'étude sur la gouvernance du foncier agro-sylvo-pastoral dans les régions de Louga, Saint-Louis et Matam. Repéré à http://www.congad.org/Rapport-de-Etude-sur-la-gouvernance-du-foncier-Agrosylvopastoral/debut/accueil.html

Dimé, M. (2017). Le mécanisme de Varsovie relatif aux pertes et préjudices liés au changement climatique : résonances et appropriations locales d'un dispositif international de compensation dans le delta du Saloum (Sénégal). Communication présentée à l'Institut sur la justice économique, CODESRIA, Harare, Zimbabwe.

Dimé M. (2009). Décentralisation et émergence d’une écocitoyenneté en milieu rural sénégalais : initiatives de prise en charge de problèmes environnementaux par l'association Fannabara à Missirah. Dans D. Ndiaye et A. Touré (dir.), Gouvernance locale et gestion décentralisée des ressources naturelles (p. 77-90). Dakar, Sénégal : Centre de suivi écologique.

Diop M.-C. (dir.). (2003). La société sénégalaise entre le local et le global. Paris, France : Karthala.

Diop, M.-C. (dir.) (2013a). Les institutions et politiques publiques à l'épreuve d'une gouvernance libérale. Paris, France : Karthala-CRES.

Diop, M.-C (dir.) (2013b). Le Sénégal sous Abdoulaye Wade. Le Sopi à l'épreuve. Paris, France : Karthala.

ENDA PRONAT (2011). Amélioration et sécurisation de l'accès des femmes au foncier au Sénégal (Projet de recherche-action participative, n 104383-001). Dakar, Sénégal : CRDI.

Fall, M. et Dimé, M. (2009). S'adapter à la dégradation de l'environnement dans le delta du Saloum : variabilité des perceptions et des pratiques chez les femmes socé et niominka. Dans D. Ndiaye et A. Touré (dir.), Gouvernance locale et gestion décentralisée des ressources naturelles (p. 301-310). Dakar, Sénégal : Centre de suivi écologique.

Fall, M. (2006). Dynamique des acteurs, conflits et modes de résolution pour une gestion durable des ressources naturelles dans la réserve de biosphère du delta du Saloum (Sénégal) (Thèse de doctorat inédite). Université de Montréal.

Fall, M. et Villeneuve, D. (2010). Gouvernance participative dans la réserve de biosphère du delta du Saloum : quelles stratégies d'acteurs? Dans C. Gagnon (dir.), L'écotourisme visité par les acteurs territoriaux. Québec, Québec : PUQ.

Falque, M., Lamotte, H. et al. (2008). Les ressources foncières : droits de propriété, économie et environnement. Cachan, France : Lavoisier.

Faye, I. M. (2011). Les acquisitions de terres à grande échelle au Sénégal : description d'un nouveau phénomène. Dakar, Sénégal : Initiative prospective agricole et rurale (IPAR).

Féral, F. (2007). L’administration des aires marines protégées en Afrique de l'Ouest. Mondes en développement, 2(138), 43-60.

Fourchard, L. (dir.) 2007. Gouverner les villes d'Afrique : État, gowvernement local et acteurs privés. Paris, France : Karthala.

Gastellu, J.-M. (1981). L'égalitarisme économique des Serer du Sénégal. Paris, France : OSTROM.

Goislard, C. et Cubrilo, O. (1998). Bibliograpbie et lexique du foncier en Afrique noire. Paris, France : Karthala.

Hathie, I., Wade, I., Ba, S., Niang, A., Niang, M., Kesso, M. S., ... Ba, C. O. (2014). Emploi des jeunes et migration en Afrique de l'Ouest (Rapport de recherche, IPAR).

INTERRESEAUX (2015). Jeunes ruraux en Afrique de l'Ouest : quelles réalités et quelles perspectives? Repéré à http://www.ired.org/modules/infodoc/cache/files/bds no17 jeunes.pdf

Koopman, J. (2012). Land grabs, government, peasant and civil society activism in the Senegal River Valley. Review of African Political Economy, 39(134), 655-664.

Kouassigan, G. A. (1966). L'Homme et la Terre, droits fonciers coutumiers et droits de propriété en Afrique occidentale. Paris, France : OSTROM. 
Lavigne Delville, P. (2009). Conceptions des droits fonciers, récits de politique publique et controverses, les plans fonciers ruraux en Afrique de l'Ouest. Dans J. P. Colin, P. Y. Le Meur et E. Leonard (dir.), Les politiques d'enregistrement des droits fonciers : du cadre légal aux pratiques locales (p. 69-103). Paris, France : Karthala.

Le Bris, E. et Le Roy, E. (1983). Enjeux fonciers en Afrique noire. Paris, France : ORSTOM, Karthala.

Niang, A., Sarr, N. F. M., Hathie, I., Diouf, N. C., Ba, C. O. et Ka, I. (2015). Comprendre les changements dans l'accès et l'utilisation de la terre par les populations rurales pawres en Afrique subsaharienne : cas du Sénégal (Rapport de recherche, IPAR, IIED, IFAD). Repéré à http://www.ipar.sn/IMG/pdf/rapport final claussa vf.pdf

Sarr, S.-M. (2013). Adaptation face aux vulnérabilités en contexte de changement climatique. Le cas de la population insulaire de Dionewar dans le delta du Saloum (Mémoire de maîtrise inédit). Université Gaston Berger de Saint-Louis.

Sénégal (2013). Plan Sénégal émergent. Repéré à www.gouv.sn/IMG/pdf/PSE.pdf

Sy, B.-A. (dir.), 2012. Changements climatiques, dynamiques des milieux et crises de sociétés en Afrique de l'Ouest, Paris, L'Harmattan.

Sy, C. B., Cissé, El, H. T. et Ba, S. (2013). Étude participative sur les acquisitions massives de terres agricoles en Afrique de l'Ouest et leur impact sur l'agriculture familiale et la sécurité alimentaire des populations locales : État des lieux. Cas du Sénégal. Coalition pour la protection du patrimoine génétique africain (COPAGEN), Inter Pares, Réseau d'études des dynamiques transnationales et de l'action collective (REDTAC).

Verdier, R. et Rochegude, A. (1995). Systèmes fonciers à la ville et au village, Paris, France : L'Harmattan.

Villeneuve, D. (2010). La gestion participative des ressources naturelles : une alternative à l'exode des jeunes du delta du Saloum (Sénégal) (Mémoire de maîtrise inédit). Université du Québec à Chicoutimi. 